\title{
A FINITE GLOBAL AZUMAYA THEOREM IN ADDITIVE CATEGORIES
}

\author{
DAVID M. ARNOLD ${ }^{1}$
}

\begin{abstract}
Let $\mathbf{C}$ be an additive category such that idempotent endomorphisms have kernels, $C$ a class of objects of $\mathbf{C}$ having Dedekind domains as endomorphism rings, and assume that if $X$ and $Y$ are quasi-isomorphic objects of $C$ then $\operatorname{Hom}(X, Y)$ is a torsion-free module over the endomorphism ring of $X$. If $A \oplus B=C_{1} \oplus \cdots \oplus C_{n}$ with each $C_{i}$ in $C$, then $A=A_{1} \oplus \cdots \oplus A_{m}$, where each $A_{j}$ is locally in $C$, and $\operatorname{End}\left(A_{j}\right) \simeq \operatorname{End}\left(C_{i}\right)$ for some $i$. The proof includes a characterization of tiled orders. Moreover, there is a "local" uniqueness for finite direct sums of objects of $C$.
\end{abstract}

Let $\mathbf{C}$ be an additive category such that idempotent endomorphisms of objects of $\mathbf{C}$ have kernels and let $C$ be a class of objects of $\mathbf{C}$ with Dedekind domains as endomorphism rings. Two objects $A$ and $B$ of $C$ are quasi-isomorphic if there are maps $f \in \operatorname{Hom}(A, B)$ and $g \in \operatorname{Hom}(B, A)$ with $f g \neq 0$. The class $C$ has the torsionfree-hom condition if whenever $A$ and $B$ are quasi-isomorphic objects of $C$ then $\operatorname{Hom}(A, B)$ is a torsion-free $E(A)$-module, where $E(A)$ is the endomorphism ring of $A$. If $A$ and $X$ are objects of $\mathbf{C}$ such that $E(A)$ and $E(X)$ are integral domains and if $P$ is a prime ideal of $E(A)$ then $A$ is isomorphic to $X$ at $P$ if there are maps $f \in \operatorname{Hom}(A, X)$ and $g \in \operatorname{Hom}(X, A)$ with $E(A)=P+E(A) g f$. An object $A$ such that $E(A)$ is a Dedekind domain is locally in $C$ if for each nonzero prime ideal $P$ of $E(A)$ there is an object $X$ in $C$ such that $A$ is isomorphic to $X$ at $P$. Finally, if $C$ is a class of objects of $\mathbf{C}$ such that $E(X)$ is a Dedekind domain for each $X$ in $C$ and if $C$ has the torsion-free-hom condition then $C$ is said to be a Dedekind class.

THEOREM I. Let $\mathbf{C}$ be an additive category such that idempotent endomorphisms have kernels, and let $C$ be a Dedekind class of objects of $\mathbf{C}$. If $A \oplus B=C_{1} \oplus \cdots \oplus C_{n}$ with each $C_{i}$ in $C$, then $A=A_{1} \oplus \cdots \oplus A_{m}$, where for each $j$ there is some $i$ with $E\left(A_{j}\right) \simeq E\left(C_{i}\right)$. In particular, each $A_{j}$ is locally in $\left\{C_{1}, \ldots, C_{n}\right\}$.

Theorem I is an example of a finite Azumaya theorem, a deduction of properties of $C_{1} \oplus \cdots \oplus C_{n}$ from conditions on the endomorphism rings of the $C_{i}$ 's [AHR1, WW1]. In this case, each direct sum decomposition has a refinement into a direct sum of indecomposables. Even though direct sum decompositions into indecomposables need not be unique, there is a "local" uniqueness.

COROllary II. Let $\mathbf{C}$ be an additive category such that idempotent endomorphisms have kernels, and let $C$ be a Dedekind class of objects of $\mathbf{C}$. If $\left\{C_{1}, \ldots, C_{m}\right\}$ and $\left\{D_{1}, \ldots, D_{n}\right\}$ are families of objects of $C$ then $C_{1} \oplus \cdots \oplus C_{m} \simeq D_{1} \oplus \cdots \oplus D_{n}$

Received by the editors May 6, 1983 and, in revised form, August 5, 1983.

1980 Mathematics Subject Classification. Primary 18E05; Secondary 15A18, 16A32, 20K40.

Key words and phrases. Additive categories, Azumaya theorem, uniqueness of direct sum decompositions, orders in simple algebras.

${ }^{1}$ Research supported, in part, by N.S.F. grant \#MCS-8003060. 
if and only if $m=n$ and for each $X$ in $\left\{C_{1}, C_{2}, \ldots, C_{m}\right\}$ and each nonzero prime ideal $P$ of $E(X)$, there is a bijection from $\left\{i \mid X\right.$ is isomorphic to $C_{i}$ at $\left.P\right\}$ to $\{j \mid$ $X$ is isomorphic to $D_{j}$ at $\left.P\right\}$.

Special cases of Theorem I and Corollary $\Pi$ are proved in [AHR1, Theorems A and $\mathrm{D}]$, wherein $C$ is assumed to be a class of objects of $\mathbf{C}$ such that $E(X)$ is a principal ideal domain for each $X$ in $C$. Results of [AHR1] are used to reduce to the homogeneous case (the $C_{i}$ 's are pairwise quasi-isomorphic) which, in turn, is a consequence of a ring theoretic theorem. This approach is in contrast to [AHR1] which uses additive category techniques for the homogeneous case.

The following notation is assumed: $D$ is a division algebra; $K$ is the center of $D$; $R$ is a Dedekind domain with quotient field $K$; and $T$ is an $R$-order in $\operatorname{Mat}_{m}(D)$ ( $T$ is an $R$-subalgebra of $\operatorname{Mat}_{m}(D), T$ is finitely generated as an $R$-module and $K T=\operatorname{Mat}_{m}(D)$, the ring of $m \times m$ matrices with entries in $D$ ).

The order $T$ is said to be a tiled order if there are nonzero orthogonal idempotents $e_{1}, \ldots, e_{m}$ of $T$ with $T=e_{1} T \oplus \cdots \oplus e_{m} T$ (e.g. see [JA1, JA2]). Note that $T$ is a tiled order if and only if there is a $K$-algebra automorphism $\phi$ of $\operatorname{Mat}_{m}(D)$ with $e_{i i} \in \phi(T)$ for each $1 \leq i \leq m$, where $e_{i i}$ is the matrix with 1 in the $i, i$ position and 0 elsewhere. Tiled orders are called graduated orders in [ZA1 and PL1].

For each nonzero prime ideal $P$ of $R$ let $R_{P}$ denote the localization of $R$ at $P$. Then $T_{P}=R_{P} \otimes_{R} T$ is an $R_{P}$-order in $\operatorname{Mat}_{m}(D)$.

The ring $T$ is semiperfect if $T / J(T)$ is artinian and each idempotent of $T / J(T)$ lifts to an idempotent in $T$, where $J(T)$ is the Jacobson radical of $T$.

The following theorem is a global version of a characterization of tiled orders over local Dedekind domains (see [JA2, PL1]).

Theorem II. Assume that $R$ is a Dedekind domain, $D$ is a division algebra, $T$ is an $R$-order in $\mathrm{Mat}_{m}(D)$, and that $T_{P}$ is semiperfect for each nonzero prime ideal $P$ of $R$. Then the following statements are equivalent.

(a) If $P$ is a nonzero prime ideal of $R$ and if $f$ is a primitive idempotent of $T_{P}$, then $f_{P} f$ is isomorphic to an $R_{P}$-order in $D$.

(b) If $e$ is a primitive idempotent of $T$ then $e T e$ is isomorphic to an $R$-order in $D$.

(c) $T$ is a tiled order.

(d) For each nonzero prime ideal $P$ of $R$,

$$
T_{P} / J\left(T_{P}\right) \simeq \operatorname{Mat}_{n_{1}}\left(D_{1}\right) \times \cdots \times \operatorname{Mat}_{n_{k}}\left(D_{k}\right),
$$

where each $D_{i}$ is a division algebra and $n_{1}+n_{2}+\cdots+n_{k} \geq m$.

Note that if $T$ is an $R$-order in $\operatorname{Mat}_{m}(K)$ then $T_{P}$ is semiperfect for each prime ideal $P$ of $R$ (e.g. [ROHD1, IV 12, p. 189]).

Theorem III includes the case that $T$ is a hereditary $R$-order in $\operatorname{Mat}_{m}(K)$. On the other hand, there is a $Z_{p}$-order in $\operatorname{Mat}_{2}(Q)$ satisfying Theorem III that is not hereditary (Example 1.2). Not every $Z$-order in $\operatorname{Mat}_{m}(Q)$ satisfies Theorem III (Example 1.3).

\section{Orders in matrix rings over division algebras.}

Proof of Theorem III. (a) $\Rightarrow$ (b) Let $e$ be a primitive idempotent of $T$ and let $P$ be a nonzero prime ideal of $R$. There is a set $\left\{e_{1}, \ldots, e_{k}\right\}$ of primitive orthogonal idempotents of $T_{P}$ with $T_{P}=e_{1} T_{P} \oplus \cdots \oplus e_{k} T_{P}, e_{i} T_{P} e_{i}$ isomorphic to an $R_{P}$-order in $D$ for each $i$, and $e=e_{1}+e_{2}+\cdots+e_{l}$. Then each $e_{i} K T_{P} e_{i} \simeq D$, so that $\operatorname{Mat}_{m}(D)=$ 
$K T_{P}=e_{1} K T_{P} \oplus \cdots \oplus e_{k} K T_{P}$ and $k=m$. Thus, there is a $K$-algebra automorphism $\phi_{P}$ of $\operatorname{Mat}_{m}(D)$ with $e_{i i} \in \phi_{P}\left(T_{P}\right)$ for each $1 \leq i \leq m$ and $\phi_{P}(e)=e_{11}+\cdots+e_{l l}$. By the Skolem-Noether theorem (e.g. [RE1]) there is a unit $u_{P}$ of $\operatorname{Mat}_{m}(D)$ with $\phi_{P}(x)=u_{P} x u_{P}^{-1}$ for each $x \in T_{P}$.

Let $f_{P}=u_{P}^{-1} e_{11} u_{P}=\phi_{P}^{-1}\left(e_{11}\right) \in T_{P}$. Then $f_{P}$ is an idempotent of $T_{P}$ and $e f_{P} e=f_{P}$, since

$$
\begin{aligned}
\phi_{P}\left(e f_{P} e\right) & =\phi_{P}(e) \phi_{P}\left(f_{P}\right) \phi_{P}(e)=\left(e_{11}+\cdots+e_{l l}\right) e_{11}\left(e_{11}+\cdots+e_{l l}\right) \\
& =e_{11}=\phi_{P}\left(f_{P}\right) .
\end{aligned}
$$

Thus, $f_{P} T_{P}$ is isomorphic to a $T_{P}$-summand of $e T_{P}$. But

$$
e_{11} T_{P}=u_{P} f_{P} u_{P}^{-1} T_{P} \simeq f_{P} u_{P}^{-1} T_{P} \simeq f_{P} T_{P}
$$

as right $T_{P}$-modules. Consequently, $e_{11} T_{P}$ is isomorphic to a $T_{P}$-module summand of $e T_{P}$ for each nonzero prime ideal $P$ of $R$. Hence, $e T=M \oplus N$ for some right $T$-modules $M$ and $N$ with $M_{P} \simeq e_{11} T_{P}$ for each nonzero prime ideal $P$ of $R$ [SE1, Corollary 6.13]. Since $e$ is primitive, $e T=M$ and $e_{11} T_{P} \simeq e T_{P}$ for each nonzero prime ideal $P$. Hence, $e T e \subseteq e T_{P} e \simeq e_{11} T_{P} e_{11} \subseteq D$, so that $e T e$ is isomorphic to an $R$-order in $D$.

(b) $\Rightarrow$ (c) As noted earlier, $T=e_{1} T \oplus \cdots \oplus e_{k} T$, with each $e_{i} T e_{i}$ isomorphic to an $R$-order in $D$, implies that $k=m$.

(c) $\Rightarrow(\mathrm{d})$ Since $T$ is a tiled order, $T_{P}$ is a tiled order, say $T_{P}=e_{1} T_{P} \oplus \cdots \oplus e_{m} T_{P}$. Then

$$
T_{P} / J\left(T_{P}\right)=\left(e_{1} T_{P}+J\left(T_{P}\right)\right) / J\left(T_{P}\right) \oplus \cdots \oplus\left(e_{m} T_{P}+J\left(T_{P}\right)\right) / J\left(T_{P}\right)
$$

is a semisimple artinian ring with each $e_{i}+J\left(T_{P}\right) \neq 0$. Hence $T_{P} / J\left(T_{P}\right)$ has at least $m$ simple summands so that $T_{P} / J\left(T_{P}\right)=\operatorname{Mat}_{n_{1}}\left(D_{1}\right) \times \cdots \times \operatorname{Mat}_{n_{k}}\left(D_{k}\right)$ with $n_{1}+\cdots+n_{k} \geq m$ and each $D_{i}$ a division algebra.

(d) $\Rightarrow$ (a) Let $P$ be a nonzero prime ideal of $R$. Since $T_{P}$ is semiperfect, there is a set of orthogonal idempotents $e_{1}, \ldots, e_{m}$ with $T_{P}=e_{1} T_{P} \oplus \cdots \oplus e_{m} T_{P}$ (lift $m$ of the diagonal idempotents from $T_{P} / J\left(T_{P}\right)$, e.g. [AF1, Proposition 27.4]). Then $e_{i} T_{P} e_{i}$ is isomorphic to an $R_{P}$-order in $D$ so that each $e_{i}$ is primitive. Thus $e_{i} T_{P} e_{i} \simeq \operatorname{End}_{T_{P}}\left(e_{i} T_{P}\right)$ is a local ring [AF1, Theorem 27.6]. Now let $f$ be a primitive idempotent of $T_{P}$. Then $f T_{P} \simeq e_{i} T_{P}$ for some $i$ (the classical local Azumaya Theorem, e.g. [AF1, Theorem 12.6]). Thus, $f T_{P} f \simeq e_{i} T_{P} e_{i}$ is isomorphic to an $R_{P}$-order in $D$ as desired.

Corollary 1.1. Assume that $R$ is a Dedekind domain with quotient field $K$ and that $T$ is an $R$-order in $\operatorname{Mat}_{m}(K)$. If $T$ is a tiled order and $e$ is a primitive idempotent of $T$ then $e T e \simeq R$.

Proof. By Theorem III, and the remark following Theorem III, $e T e$ is isomorphic to an $R$-order $S$ in $K$. Since $R$ is Dedekind and $R \subseteq S \subseteq K$, the quotient field of $R$, it follows that $R \simeq e T e$.

EXAMPLE 1.2. Given a prime $p$ of $Z$,

$$
T=\left(\begin{array}{cc}
Z & Z \\
p^{2} Z & Z
\end{array}\right)
$$

is a $Z$-order in $\operatorname{Mat}_{2}(Q)$ such that if $e$ is a primitive idempotent of $T$ then $e T e \simeq Z$ but $T$ is not a hereditary $Z$-order in $\operatorname{Mat}_{2}(Q)$. 
ExAmple 1.3. Let $T=\left\{\left(\begin{array}{ll}a & b \\ p c & d\end{array}\right) \mid a \equiv d(\bmod p), a, b, c, d \in Z_{p}\right\}$. Then $T=T_{p}$ is semiperfect, 1 is a primitive idempotent in $T$, and $T$ is not isomorphic to $Z_{p}$.

\section{A finite global Azumaya theorem.}

LEMMA 2.1. Let $G=C_{1} \oplus \cdots \oplus C_{m}$, where the $C_{i}$ 's are pairwise quasi-isomorphic objects of a Dedekind class $C$. Then there is a Dedekind domain $R$ with quotient field $K$ and an $R$-order $T$ in $\operatorname{Mat}_{m}(K)$ such that each $E\left(C_{i}\right)$ may be identified with $R, E(G) \simeq T$, and $T$ is a tiled order.

Proof. A consequence of [AHR1, Theorems 2.1, 2.2 and Corollary 2.3].

LEMMA 2.2. Let $\mathbf{C}$ be an additive category such that idempotent endomorphisms of objects of $\mathbf{C}$ have kernels, and let $C$ be a Dedekind class of objects of $\mathbf{C}$. The following are equivalent.

(a) If $G=A \oplus B=C_{1} \oplus \cdots \oplus C_{n}$ with each $C_{i}$ in $C$ then $A=A_{1} \oplus \cdots \oplus A_{m}$, where for each $j$ there is some $i$ with $E\left(A_{j}\right) \simeq E\left(C_{i}\right)$.

(.b) If $G=A \oplus B=C_{1} \oplus \cdots \oplus C_{n}$ with each $C_{i}$ in $C$ and the $C_{i}$ 's are pairwise quasi-isomorphic then $A=A_{1} \oplus \cdots \oplus A_{m}$, where for each $j$ there is some $i$ with $E\left(A_{j}\right) \simeq E(C i)$.

(c) If $G=C_{1} \oplus \cdots \oplus C_{n}$ with $C_{i}$ in $C$, the $C_{i}$ 's are pairwise quasi-isomorphic, and $e$ is a primitive idempotent in $E(G)$, then $e E(G) e \simeq E\left(C_{i}\right)$ for some $i$.

Proof. (a) $\Rightarrow(\mathrm{b})$ is clear.

(b) $\Rightarrow$ (a) Group the $C_{i}$ 's by quasi-isomorphism classes to get $G=A \oplus B=K_{1} \oplus$ $\cdots \oplus K_{l}$. Then $A=A_{1} \oplus \cdots \oplus A_{l}$, where each $A_{i}$ is a summand of $K_{i}$ [AHR1, Corollary 2.6]. Now apply (b).

(b) $\Rightarrow(\mathrm{c})$ Note that $G=e(G) \oplus(1-e)(G)$, where $e(G)=\operatorname{Kernel}(1-e)$ and $(1-e)(G)=\operatorname{Kernel}(e)$. Since $e$ is primitive, $e(G)$ is indecomposable so that $e E(G) e \simeq$ $E(e(G)) \simeq E\left(C_{i}\right)$ for some $i$.

(c) $\Rightarrow$ (b) If $A_{1}$ is an indecomposable summand of $A$ then $A_{1}=e(G)$ for some primitive idempotent $e$ of $E(G)$. Thus $E\left(A_{1}\right) \simeq E(e(G)) \simeq e E(G) e \simeq E\left(C_{i}\right)$ for some $i$. Finally, by Lemma 2.1, there is a Dedekind domain $R$ with $R \simeq E\left(C_{i}\right)$ for each $i$ and $E(G)$ is a finite rank torsion-free $R$-module. Consequently, $A=A_{1} \oplus \cdots \oplus A_{m}$ with each $A_{j}$ indecomposable. Therefore, $E\left(A_{j}\right) \simeq R \simeq E\left(C_{i}\right)$ for each $j$.

Proof of Theorem I. Assume that $A \oplus B=C_{1} \oplus \cdots \oplus C_{n}$ with each $C_{i}$ in $C$. By Lemma 2.2, it is sufficient to assume that the $C_{i}$ 's are pairwise quasi-isomorphic. Now apply Lemma 2.1, Corollary 1.1 , and Lemma 2.2 to see that $A=A_{1} \oplus \cdots \oplus A_{n}$, where for each $j$ there is some $i$ with $E\left(A_{j}\right) \simeq E\left(C_{i}\right)$, a Dedekind domain. Let $X=$ $A_{j}$. There are maps $f_{k} \in \operatorname{Hom}\left(X, C_{k}\right)$ and $g_{k} \in \operatorname{Hom}\left(C_{k}, X\right)$ with $1_{X}=g_{1} f_{1}+\cdots+$ $g_{n} f_{n}$, since $X$ is a summand of $C_{1} \oplus \cdots \oplus C_{n}$. If $P$ is a nonzero prime ideal of $E(X)$ then some $g_{k} f_{k} \notin P$. Thus, $E(X)=P+E(X) g_{k} f_{k}$ and $X=A_{j}$ is locally in $\left\{C_{1}, C_{2}, \ldots, C_{n}\right\}$.

Proof of Corollary II. $(\Rightarrow)$ Assume that

$$
G=C_{1} \oplus \cdots \oplus C_{m} \simeq D_{1} \oplus \cdots \oplus D_{n}
$$

with each $C_{i}$ and $D_{j}$ in $C$. It suffices to assume that the $C_{i}$ 's and $D_{j}$ 's are all quasiisomorphic by grouping together the $C_{i}$ 's and $D_{j}$ 's in quasi-isomorphism classes and applying [AHR1, Corollary 2.6 and Theorem 2.7]. By Lemma 2.2, there is a Dedekind domain $R$ with quotient field $K$ and an $R$-order $T$ in $\operatorname{Mat}_{m}(K)$ such that $E\left(C_{i}\right)=R$ for each $i, E(G) \simeq T$, and $T$ is a tiled order. 
If $P$ is a nonzero prime ideal of $R$ then $R_{P}$ is a local ring and $T_{P}$ is an $R_{P}$-order in $\operatorname{Mat}_{m}(K)$ with a full set of idempotents. Choose idempotents $e_{i}$ and $f_{j}$ of $T$ such that $1=e_{1}+\cdots+e_{m}=f_{1}+\cdots+f_{n}, C_{i} \simeq e_{i} G=\operatorname{Kernel}\left(1-e_{i}\right)$, and $D_{j} \simeq f_{j} G=$ $\operatorname{Kernel}\left(1-f_{j}\right)$. Then $E\left(D_{j}\right) \simeq f_{j} T f_{j} \simeq R$, by Theorem I, since $E\left(D_{j}\right)$ a domain implies that $f_{j}$ is primitive. Hence, $m=n$.

Now $T_{P}=e_{1} T_{P} \oplus \cdots \oplus e_{m} T_{P}=f_{1} T_{P} \oplus \cdots \oplus f_{m} T_{P}$ and $E\left(e_{i} T_{P}\right) \simeq R_{P} E\left(f_{j} T_{P}\right)$ is a local ring. By the classical local Azumaya theorem, there is a permutation $\sigma$ of $\{1,2, \ldots, m\}$ such that $e_{i} T_{P} \simeq f_{\sigma(i)} T_{P}$ for each $i$. Thus, there is

$$
h_{i}^{\prime}: e_{i} T \rightarrow f_{\sigma(i)} T \text { and } g_{i}^{\prime}: f_{\sigma(i)} T \rightarrow e_{i} T
$$

with $E\left(e_{i} T\right)=P+E\left(e_{i} T\right) g_{i}^{\prime} h_{i}^{\prime}$, noting that $E\left(e_{i} T\right)=e_{i} T e_{i}=E\left(C_{i}\right)=R$. Then

$$
h_{i}=f_{\sigma(i)} h_{i}^{\prime} e_{i}: C_{i} \rightarrow D_{\sigma(i)} \text { and } g_{i}=e_{i} g_{i}^{\prime} f_{\sigma(i)}: D_{\sigma(i)} \rightarrow C_{i}
$$

with $E\left(C_{i}\right)=P+E\left(C_{i}\right) g_{i} h_{i}$. Consequently, $\sigma$ induces a bijection from $\{i \mid X$ is isomorphic to $C_{i}$ at $\left.P\right\}$ to $\left\{j \mid X\right.$ is isomorphic to $D_{j}$ at $\left.P\right\}$.

$(\Leftarrow)$ It suffices to assume that the $C_{i}^{\prime}$ 's and $D_{j}^{\prime}$ 's are all quasi-isomorphic. By Lemma 2.2, there is a Dedekind domain $R$ with $E\left(D_{j}\right)=R$ for each $j$. Since $C_{1}$ is locally in $\left\{D_{1}, D_{2}, \ldots, D_{n}\right\}$,

$$
D_{1} \oplus \cdots \oplus D_{n}=C_{1} \oplus B_{2} \oplus \cdots \oplus B_{k}
$$

for some $B_{i}$ (as in [AHR1, Lemma 3.1]). By Theorem I, it suffices to assume that $E\left(B_{i}\right)=R$ for $2 \leq i \leq k$ and that each $B_{i}$ is locally in $\left\{D_{1}, D_{2}, \ldots, D_{n}\right\}$. As a consequence of $(\Rightarrow)$ and the hypothesis, for each $X$ in $\left\{C_{1}, C_{2}, \ldots, C_{m}\right\}$ and each prime ideal $P$ of $E(X)$ there is a bijection from $\left\{i \neq 1 \mid X\right.$ is isomorphic to $C_{i}$ at $\left.P\right\}$ to $\left\{j \mid X\right.$ is isomorphic to $B_{j}$ at $\left.P\right\}$. By induction on the number of $C_{i}^{\prime}$ s, $C_{2} \oplus \cdots \oplus$ $C_{m} \simeq B_{2} \oplus \cdots \oplus B_{k}$ and so $C_{1} \oplus \cdots \oplus C_{m} \simeq D_{1} \oplus \cdots \oplus D_{n}$, as desired.

REMARK. Applications of Theorem I and Corollary II include the applications listed in [AHR1, §6], with appropriate and obvious modifications, the finite rank case of [KO1, Theorem 8], and a generalization of [AR1, Theorem 6.11].

\section{REFERENCES}

[AF1] F. W. Anderson and K. R. Fuller, Rings and categories of modules, Graduate Texts in Math., vol. 13, Springer-Verlag, New York, 1970.

[AHR1] D. Arnold, R. Hunter and F. Richman, Global Azumaya theorems in additive categories, J. Pure Appl. Algebra 16 (1980), 223-242.

[AR1] D. M. Arnold, Finite rank torsion free abelian groups and rings, Lecture Notes in Math., vol. 931, Springer-Verlag, New York, 1980.

[JA1] V. A. Jategaonkar, Global dimension of tiled orders over a discrete valuation ring, Trans. Amer. Math. Soc. 196 (1974), 313-330.

[JA2] , Global dimension of tiled orders over commutative domains, Trans. Amer. Math. Soc. 190 (1974), 357-374.

[KO1] G. Kolettis, Jr., Homogeneous completely decomposable modules, Studies on Abelian Groups, Dunod, Paris, 1968, pp. 223-238.

[PL1] W. Plesken, Group rings of finite groups over $p$-adic integers, Lecture Notes in Math., SpringerVerlag, New York (to appear).

[RE1] I. Reiner, Maximal orders, Academic Press, New York, 1975.

[RO1] K. W. Roggenkamp, Lattices over orders. II, Lecture Notes in Math., vol. 142, SpringerVerlag, Berlin, 1970.

[ROHD1] K. W. Roggenkamp and V. Huber-Dyson, Lattices over orders. I, Lecture Notes in Math., vol. 115, Springer-Verlag, Berlin, 1970. 
[SE1] R. G. Swan and E. G. Evans, $K$-theory of finite groups and orders, Lecture Notes in Math., vol. 149, Springer-Verlag, Berlin, 1970.

[WW1] C. L. Walker and R. B. Warfield, Jr., Unique decompositions and isomorphic refinement theorems in additive categories, J. Pure Appl. Algebra 7 (1976), 347-359.

[ZA1] H. Zassenhaus, Graduated orders, preprint.

Department of Mathematical Sciences, New Mexico State University, Las Cruces, NEW MeXICO 88003 